\title{
Direct detection data and possible hints for low-mass WIMPs
}

\section{Thomas Schwetz*}

Max-Planck-Institute for Nuclear Physics, PO Box 103980, 69029 Heidelberg, Germany

E-mail: schwetz AT mpi-hd.mpg.de

Possible hints for WIMP dark matter with mass around $10 \mathrm{GeV}$ coming from the DAMA, CoGeNT, and maybe also CRESST experiments are presented, and confronted with constraints from CDMS and XENON data. Focusing on spin-independent (SI) WIMP-nucleus interactions, I elaborate on the difficulties to make the hints consistent with each other and to evade the constraints, mentioning energy scale uncertainties, quenching and light-yield factors, as well as uncertainties on halo properties. In the present situation it seems hard to reconcile all data within the SI framework, which suggests that if the experimental anomalies were indeed due to dark matter a more exotic mechanism (to be identified) had to be at work.

Identification of Dark Matter 2010

July 26 - 302010

University of Montpellier 2, Montpellier, France

\footnotetext{
*Speaker.
} 
The dark matter direct detection experiments DAMA, CoGeNT, and maybe also CRESST report some anomalies which can be interpreted in terms of spin-independent (SI) scattering of WIMP dark matter particles with a mass around $10 \mathrm{GeV}$ and a WIMP-nucleon scattering cross section of order $10^{-40} \mathrm{~cm}^{2}$. This interpretation is challenged by constraints mainly from the CDMS and XENON experiments. The situation is summarized in Fig. 1.

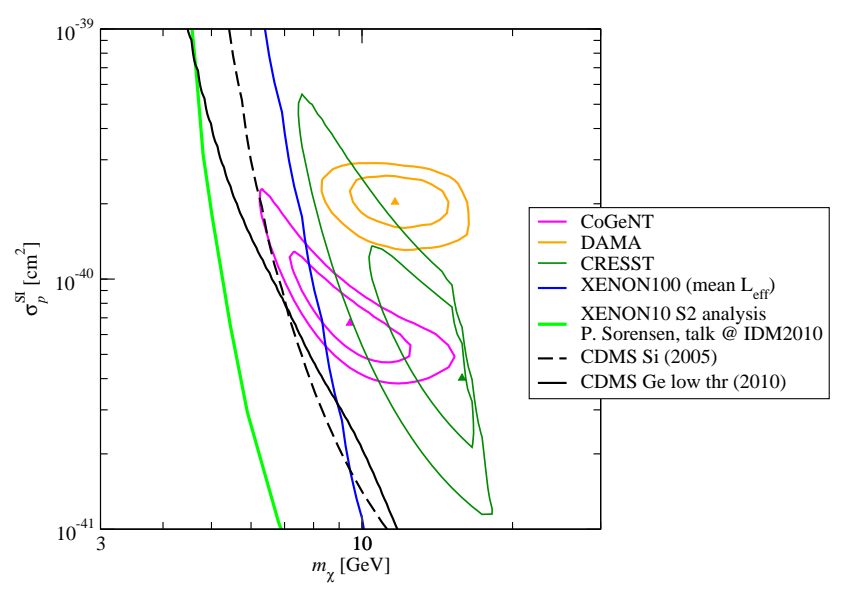

Figure 1: Hints from DAMA, CoGeNT, and CRESST at 90\% CL and $3 \sigma$ compared to the constraints from CDMS and XENON at $90 \%$ CL for elastic spin-independent scattering.

The hints. The DAMA experiment reports evidence at about $8.9 \sigma$ for an annual modulation of their scintillation signal from $\mathrm{NaI}$ crystals in the low energy region of the energy window, between 2 and 6 keVee [1]. The phase of the modulation is in agreement with the expectation from WIMP scattering due to the motion of the earth around the sun. Following [2], we assume that channeling is negligible for nuclear recoils, which implies that quenching has to be taken into account, i.e. only a certain fraction of the recoil energy is deposited in scintillation light. The default values are $q_{\mathrm{Na}}=0.3$ and $q_{\mathrm{I}}=0.09$ for sodium and iodine recoils, respectively. The DAMA signal can be explained in terms of SI scattering on either $\mathrm{Na}$ or I. However, the scattering on iodine requires WIMP masses of order $70 \mathrm{GeV}$ and cross sections excluded by CDMS and XENON by more than two orders of magnitude. Therefore, we focus here on scattering on $\mathrm{Na}$, which, due to its smaller nuclear mass is sensitive to lighter WIMPs, in the $10 \mathrm{GeV}$ region. The corresponding parameter region is shown by the orange contours in Fig. 1.

CoGeNT is a Ge detector with a very low threshold of 0.4 keVee and excellent energy resolution. In [3] they report an event excess between the threshold and 3.2 keVee with an exponential shape, which cannot be explained by known background sources and has a shape consistent with a signal from WIMPs with a mass around $10 \mathrm{GeV}$. The CoGeNT region shown in Fig. 1 has been obtained under the assumption that in the signal region only identified peaks and a flat background are present, apart from the WIMP signal. In particular, it has been assumed that there is no background component with exponential shape.

The CRESST-II experiment searches for WIMP recoils in a $\mathrm{CaWO}_{4}$ target [4]. Using the relative signal in light and phonons it is possible to distinguish nuclear recoil events from $\mathrm{W}$ and $\mathrm{O}$ as well as $\alpha$ or $\gamma$ background events. At this conference an unexplained excess of events in the $\mathrm{O}$ band has been reported [4]. A total of 32 events has been observed with an expected background 

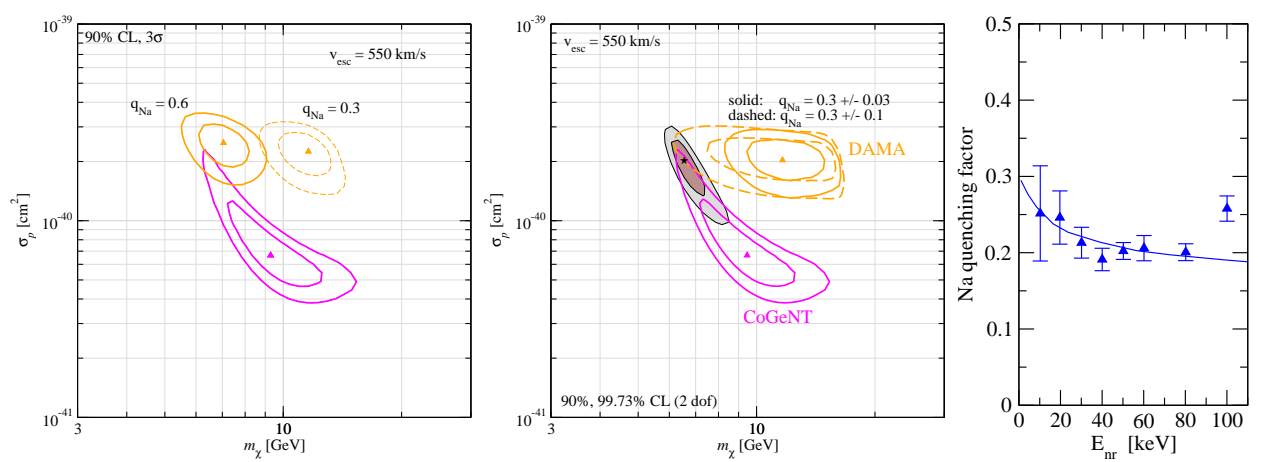

Figure 2: Left: DAMA region compared to CoGeNT for two different assumptions on the Na quenching factor, $q_{\mathrm{Na}}=0.3$ (dashed) and $q_{\mathrm{Na}}=0.6$ (solid). Middle: DAMA regions marginalizing over the uncertainty on $q_{\mathrm{Na}}$ for two assumptions on the error. The shaded region corresponds to a combined DAMA/CoGeNT fit for $q_{\mathrm{Na}}=0.3 \pm 0.1$. Right: measurement of $q_{\mathrm{Na}}$ from [7].

of $8.4 \pm 1.4$ events, mainly from $\alpha$ 's with a small contribution from neutrons and $\gamma$ 's, see [5] for a recent update confirming the excess. We have performed a rough estimate of the region in WIMP mass and SI cross section which could account for the signal by using the information given in [4]. The region shown in Fig. 1 assumes $400 \mathrm{~kg}$ d exposure at 100\% efficiency and uses the distribution of signal as well as background events within the various CRESST detectors. The region is cut off for $m_{\chi} \gtrsim 20 \mathrm{GeV}$ by requiring that the signal is consistent with the bound from the $\mathrm{W}$ band given in [4] for the inelastic scattering scenario. While this result is highly speculative and has to await confirmation from the CRESST collaboration it is intriguing that the region ends up in the same ballpark as the DAMA and CoGeNT hints. Given the still unconfirmed nature of the CRESST signal we focus in the following on the DAMA and CoGeNT results.

From Fig. 1 it follows that although DAMA and CoGeNT indicate similar WIMP masses, they require cross sections different by a factor 2 to 5. In [6] it has been suggested that uncertainties in the quenching factor for $\mathrm{Na}$ could reconcile the two results. Fig. 2 (left) shows that for larger $q_{\mathrm{Na}}$ the DAMA region shifts to smaller WIMP masses which potentially could make it consistent with the CoGeNT region. The DAMA regions in the middle panel have been obtained by assuming a central value of $q_{\mathrm{Na}}=0.3$ but allowing to float it in the fit by adding a Gaussian penalty function to the $\chi^{2}$ assuming an error of \pm 0.03 (solid contours) or \pm 0.1 (dashed contours). In the latter case marginal overlap is found with CoGeNT which might allow a combined fit (shaded region). The minimum $\chi^{2}$ of the combined fit (assuming $\left.q_{\mathrm{Na}}=0.3 \pm 0.1\right)$ is $\chi_{\mathrm{comb}}^{2}=75 /(68-4)$ corresponding to a probability of $1.6 \%$. In contrast, if each experiment is fitted separately very good fits are obtained: $\chi_{\text {DAMA }}^{2}=8.2 /(12-2),(61 \%)$ and $\chi_{\text {CoGeNT }}^{2}=46 /(56-4),(71 \%)$. This indicates that sever tension remains in the combined fit. In the right panel we show a recent measurment of $q_{\mathrm{Na}}$ from [7]. These data suggest even a slightly lower value of $q_{\mathrm{Na}}$ in the low energy region relevant for DAMA $\left(E_{n r} \simeq 10 \mathrm{keV}\right.$ ) of about $q_{\mathrm{Na}} \approx 0.25 \pm 0.05$. From this result it seems unlikely that the uncertaintly on $q_{\mathrm{Na}}$ alone can make DAMA and CoGeNT consistent with each other. Note also that shifting the energy scale for CoGeNT due to uncertainties in the Ge quenching factors at these low energies could move the region towards DAMA. However, background peaks in the signal region at known possitions can be used to calibrate the CoGeNT energy scale. 

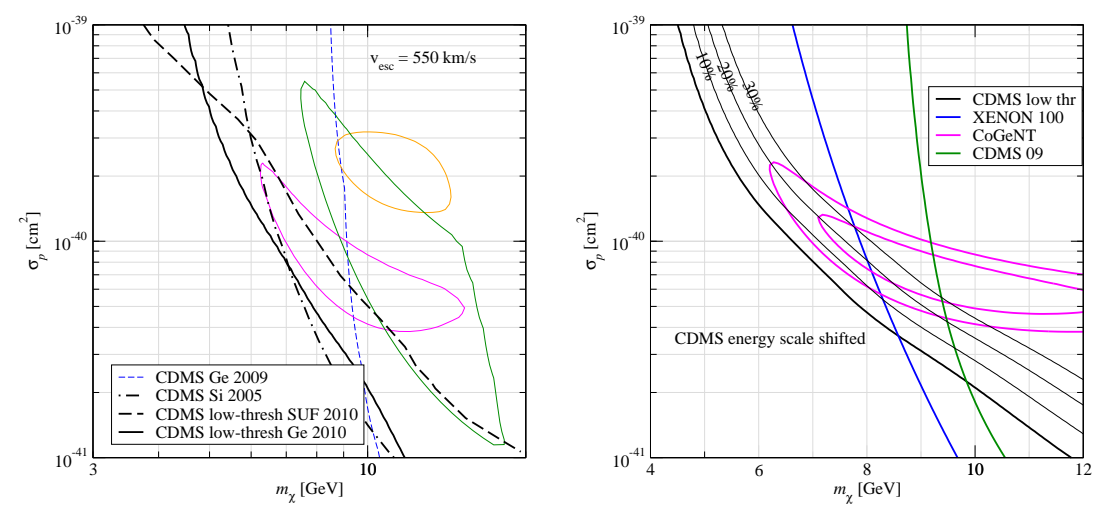

Figure 3: Left: constraints from different CDMS analyses: Ge 2009 [8], Si 2005 [9], low-thresh SUF 2010 [10], and low-thresh Ge 2010 [11]. Right: effect of a wrongly calibrated energy scale in the low-threshold Ge 2010 analysis. The thin black curves correspond to an energy scale shifted by $10 \%, 20 \%$, and $30 \%$.

Constraints. The non-observation of a significant excess of events in the CDMS experiment [8] is a challenge for the dark matter interpretation of these anomalies in terms of SI interactions. Fig. 3 (left) shows the bounds from CDMS coming from different analyses. The "standard" CDMS result labeled "CDMS Ge 2009" is based on a $10 \mathrm{keV}$ threshold applying usual nuclear recoil selection cuts, yielding 2 candidate events over an expected background of $0.8 \pm 0.22$ [8]. Other analyses are less sensitive in the more conventional WIMP region $m_{\chi} \gtrsim 50 \mathrm{GeV}$, but can provide stronger constraints on low-mass WIMPs. The "Si 2005" analysis [9] is based on $12 \mathrm{~kg}$ d silicon data with a $7 \mathrm{keV}$ threshold. Thanks to the lower mass of $\mathrm{Si}$ compared to $\mathrm{Ge}$ and the slightly lower threshold these data essentially exclude the CoGeNT/DAMA region. This result is enforced by the recent "low-threshold Ge 2010" analysis [11], where cuts on nuclear recoil/electron event discrimination are relaxed accepting some background but allowing to lower the threshold to $2 \mathrm{keV}$.

An important issue in interpreting such bounds is the calibration of the energy scale of the detector, since the limit is dominated by the energy threshold, where the largest signal is expected. Fig. 3 (right) illustrates how a hypothetical error in the energy scale calibration would affect the limit from the low-threshold Ge 2010 analysis. It can be seen that only a major shift in the energy scale of order $30 \%$ can sufficiently relax the bound. To the author it seems unlikely that such a large mis-calibration happened, especially since the predicted/extrapolated background matches well the observed event spectrum, see Fig. 1 of [11].

Let us now move to the results from the XENON10 [12] and XENON100 [13] experiments, which also provide serious constraints in the region of interest. Using a coincidence in signals from scintillation ( $\mathrm{S} 1$ ) and ionization (S2) an efficient nuclear recoil identification is possible. The energy scale for nuclear recoils is set by the $\mathrm{S} 1$ signal, which has to be translated into nuclear recoil energy $E_{n r}$ with the help of the light-yield function $L_{\mathrm{eff}}\left(E_{n r}\right)$. Measurments of $L_{\text {eff }}$ are shown in the upper left panel of Fig. 4. For the low-mass region especially the low energy region of $L_{\text {eff }}$ is important where data are scarce and partially inconsistent. Therefore we adopt three representative curves shown in the figure. As illustrated in the lower left panel the behaviour of $L_{\text {eff }}$ is crucial to constrain low mass WIMPs, since the effective acceptance window depends on this choice, see also [14]. The corresponding limits are shown in the right panel of Fig. 4. It is clear that 

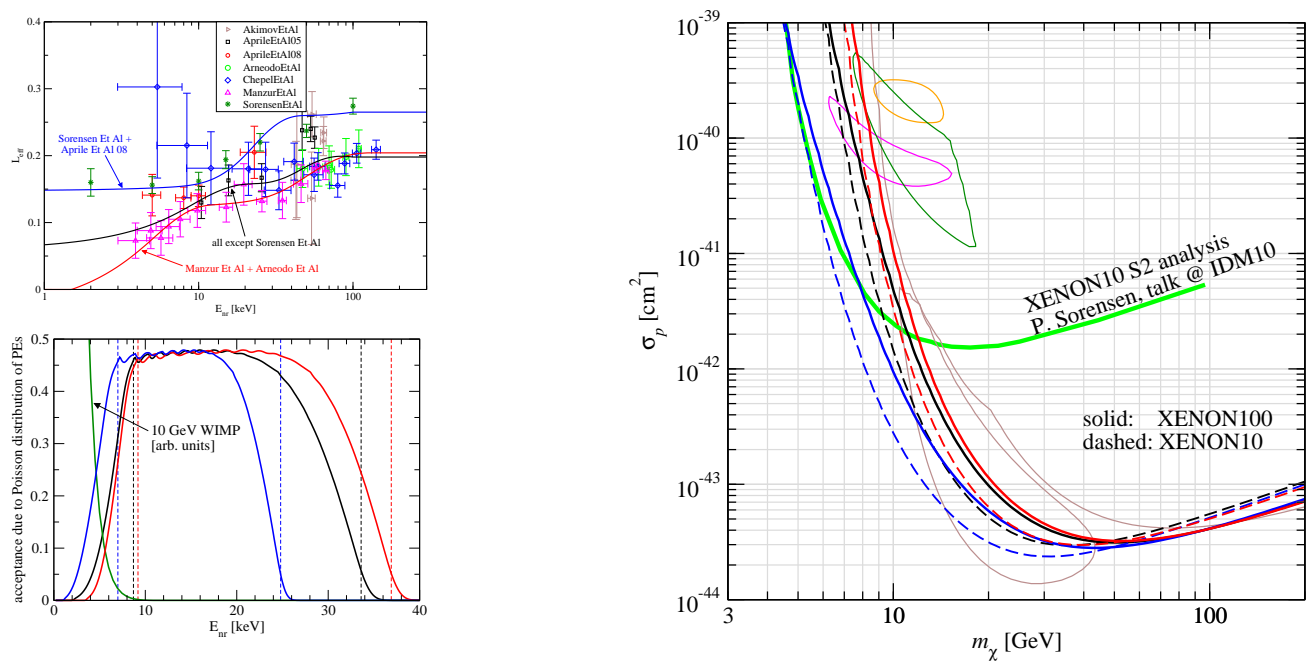

Figure 4: Upper left: compilation of data on $L_{\text {eff }}$ and three representative fits to part of the data. Lower left: the acceptance window in XENON100 for the three examples of $L_{\text {eff }}$ compared to the signal expected from a $10 \mathrm{GeV}$ WIMP. Right: exclusion curves from XENON10 (dashed) and XENON100 (solid) for the three examples of $L_{\text {eff }}$. The color of the curves in the three panels indicate the corresponding choice for $L_{\text {eff. }}$. In the right panel also the constraint from an S2-only analysis of XENON10 data [16] is shown.

if $L_{\text {eff }}$ is not too low in the low energy region XENON10/100 results provide severe constraints on the CoGeNT/DAMA region. A recent work [15] exploring the correlation of scintillation and ionization signals in xenon suggests $L_{\text {eff }}$ values somewhere between the black and the blue curves in Fig. 4, enforcing the xenon constraints.

At this conference a preliminary analysis of XENON10 data has been presented, based only on the S2 (ionization) signal [16]. With this method background rejection is less efficient, giving a significantly weaker limit for $m_{\chi} \gtrsim 20 \mathrm{GeV}$. However, due to the larger signal in S2 compared to S1 the threshold can be lowered, allowing to put stronger constraints on low-mass WIMPs independent of the $L_{\text {eff }}$ ambiguities. As illustrated in Fig. 4 this analysis clearly excludes the region relevant for CoGeNT/DAMA.

Given this situation it seems difficult to obtain a consistent interpretation of all data in terms of SI interactions. This would require $(i)$ a major problem in the $\mathrm{Na}$ and/or Ge quenching factor determinations to make DAMA and CoGeNT consistent, (ii) a major calibration error in CDMS (for Ge [11] and Si [9] data), (iii) a major problem with the XENON S2-only analysis [16], (iv) very low values of $L_{\text {eff }}$ in Xenon in the $E_{n r} \sim$ few $\mathrm{keV}$ region.

Can a modified dark matter halo reconcile the data? The results obtained in [17] on previous data suggest that only quite extreem (possibly unrealistic) assumptions on the dark matter velocity distribution may lead to a slight improvement of the consistency of the data, for example a highly anisotropic velocity distribution. In Fig. 5 we illustrate-as two examples-effects related to the galactic escape velocity. The left panel shows the effect of changing the value of the escape velocity. Decreasing $v_{\mathrm{esc}}$ from 700 to $550 \mathrm{~km} / \mathrm{s}$ can slightly improve the consistency, since the allowed regions are practially unchanged whereas the limits move slightly to larger $m_{\chi}$. In contrast, when $v_{\text {esc }}$ is lowered even further also the DAMA/CoGeNT regions start to move to the right, and hence compatibility cannot be improved much further. The default assumption adopted in all other 

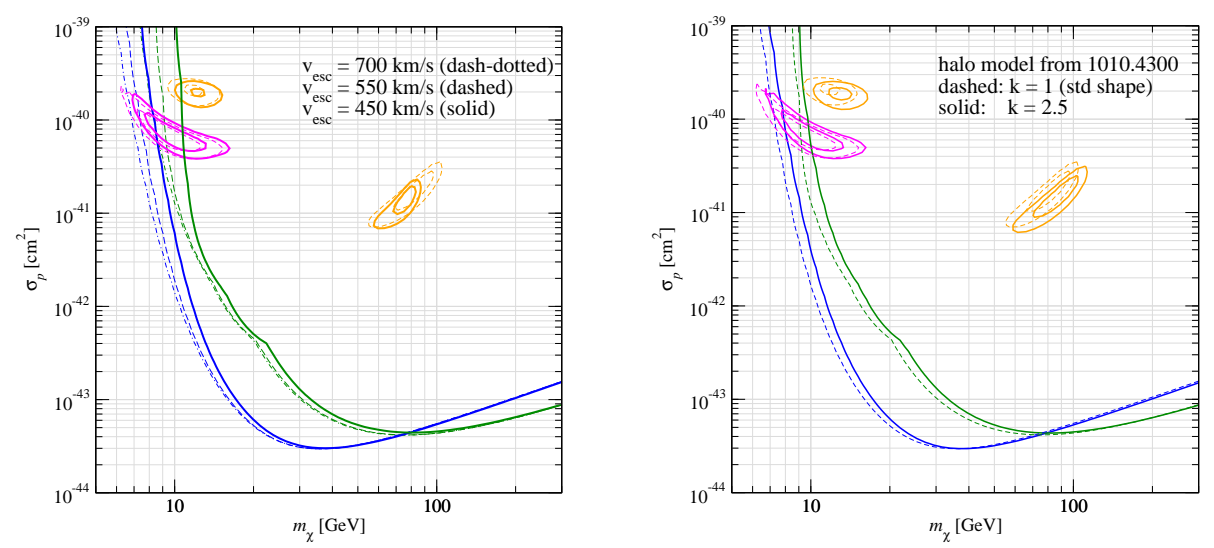

Figure 5: DAMA (orange) and CoGeNT (magenta) regions at $90 \% \mathrm{CL}$ and $3 \sigma$ compared to the $90 \% \mathrm{CL}$ exclusion curves from XENON10 (blue) and CDMS Ge 2009 (green). Left: the effect of changing the value of the galactic escape velocity. Right: changing the shape of the dark matter velocity distribution close to $v_{\text {esc }}$ according to [18].

figures shown here is $v_{\mathrm{esc}}=550 \mathrm{~km} / \mathrm{s}$. In the right panel we investigate the impact of the shape of the velocity distribution close to the cut-off as discussed recently in [18]. Our standard assumption, a Maxwellian distribution truncated at $v_{\text {esc }}$ corresponds to the parameter $k=1$ from [18], while $k=2.5$ describes a distribution going smoothly to zero with a shape motivated by the dynamics of the outer part of the galaxy. We observe that this modified distribution leads to a shift of the regions as well as the constraints, without improving significantly the relative compatibility. See also [19] on this topic.

Beyond elastic SI interactions. Let us comment briefly on some selected alternative particle physics models (without the ambition of being complete). Assuming spin-dependent (SD) interactions it is possible to use the fact that I and $\mathrm{Na}$ in DAMA have an odd number of protons, whereas $\mathrm{Xe}$ and Ge have an even number of protons. Assuming that dark matter interacts with the proton spin one can therefore evade the bounds for DAMA, though no consistent explanation for CoGeNT is obtained in this way. However, there are sever bounds from experiments using a florine target, such as COUPP [20] or PICASSO [21], which exclude the DAMA region at 90\% CL [22]. Moreover, in such a scenario neutrino constraints from the sun are tight [23], and under model dependent assumptions also collider constraints rule out such a solution, e.g. [24].

If dark matter scatters inelastically to a slightly heavier dark particle, annual modulation can be enhanced compared to the unmodulated rate, with a different spectral shape, favouring heavy targets [25]. This scenario (assuming SI interactions) has been invoked to explain DAMA (it cannot explain CoGeNT), but has been recently excluded by the $\mathrm{W}$ data from CRESST [4]. A possible way out has been proposed in [22] by assuming SD inelastic scattering on protons. This provides a valid explanation of DAMA, avoiding constraints from $\mathrm{Ge}, \mathrm{Xe}, \mathrm{W}$, since these elements have an even number of protons, as well as constraints from $F$ experiments using the inelasticity to suppress scattering on the light florine nucleus. Also in this scenario neutrino constraints are important [26], and in a given model one may expect also collider constraints to be relevant due to the relatively large cross section needed. A possible realization of SD inelastic interactions could originate from tensorial interactions [22]. A related model is discussed in [27], where dark matter interacts in- 
elastically with nuclei via the magnetic moment, using the exceptionally large magnetic moment of iodine to explain the DAMA signal.

Finally, it is intriguing that DAMA as well as CoGeNT do not discriminate between nuclear recoil and electronic events, whereas most other experiments do. The assumption that dark matter interacts only with electrons but not directly with quarks has been investigated in [28], coming, however, to a negative conclusion concerning a valid explanation of all data.

Acknowledgements. I would like to thank the IDM organizers for a very pleasant and stimulating conference. I am grateful to J. Kopp and J. Zupan for collaboration and lots of discussion on this topic. This work was partly supported by the Transregio Sonderforschungsbereich TR27 "Neutrinos and Beyond" der Deutschen Forschungsgemeinschaft.

\section{References}

[1] R. Bernabei et al. [DAMA Collaboration], Eur. Phys. J. C 56 (2008) 333 [arXiv:0804.2741]; P. Belli, these proceedings.

[2] N. Bozorgnia, G. B. Gelmini and P. Gondolo, JCAP 1011, 019 (2010) [arXiv:1006.3110]; G. Gelmini, these proceedings.

[3] C. E. Aalseth et al. [CoGeNT collaboration], arXiv:1002.4703 [astro-ph.CO].

[4] W. Seidel, these proceedings.

[5] Talk by F. Pröbst at "Dark Matter: Direct Detection and Theoretical Developments", Princeton, 15-16 November 2010, http: / / phy-gzk. princeton.edu/DMworkshop/Franz_Probst.pdf

[6] D. Hooper, J. I. Collar, J. Hall and D. McKinsey, arXiv:1007.1005 [hep-ph].

[7] H. Chagani et al., JINST 3, P06003 (2008) [arXiv:0806.1916].

[8] Z. Ahmed et al. [The CDMS-II Collaboration], Science 327, 1619 (2010) [arXiv:0912.3592]; T. Saab, these proceedings.

[9] D. S. Akerib et al. [CDMS Collaboration], Phys. Rev. Lett. 96, 011302 (2006) [astro-ph/0509259].

[10] D. S. Akerib et al. [CDMS Collaboration], arXiv:1010.4290 [astro-ph.CO].

[11] Z. Ahmed et al. [CDMS-II Collaboration], arXiv:1011.2482 [astro-ph.CO].

[12] J. Angle et al. [XENON Collaboration], Phys. Rev. Lett. 100 (2008) 021303 [arXiv:0706.0039]; Phys. Rev. D 80, 115005 (2009) [arXiv:0910.3698].

[13] E. Aprile et al. [XENON100 Collaboration], Phys. Rev. Lett. 105, 131302 (2010) [arXiv:1005.0380]; M. Schumann, these proceedings.

[14] C. Savage et al., arXiv:1006.0972 [astro-ph.CO]; C. Savage, these proceedings.

[15] F. Bezrukov, F. Kahlhoefer and M. Lindner, arXiv:1011.3990 [astro-ph.IM].

[16] P. Sorensen, these proceedings.

[17] M. Fairbairn, T. Schwetz, JCAP 0901, 037 (2009) [arXiv:0808.0704].

[18] M. Lisanti, L. E. Strigari, J. G. Wacker and R. H. Wechsler, arXiv:1010.4300 [astro-ph.CO].

[19] P. J. Fox, J. Liu and N. Weiner, arXiv:1011.1915 [hep-ph].

[20] E. Behnke et al. [COUPP Collaboration], Science 319 (2008) 933-936 [arXiv:0804.2886].

[21] S. Archambault et al. [PICASSO Coll.], Phys. Lett. B682 (2009) 185-192 [arXiv:0907.0307].

[22] J. Kopp, T. Schwetz, J. Zupan, JCAP 1002 (2010) 014 [arXiv:0912.4264].

[23] S. Desai et al. [Super-Kamiokande Coll.], Phys. Rev. D70 (2004) 083523 [hep-ex/0404025].

[24] J. Goodman et al., arXiv:1005.1286; Y. Bai, P. J. Fox, R. Harnik, arXiv:1005.3797.

[25] D. Tucker-Smith, N. Weiner, Phys. Rev. D64 (2001) 043502 [hep-ph/0101138].

[26] J. Shu, P. Yin, S. Zhu, Phys. Rev. D81 (2010) 123519 [arXiv:1001.1076].

[27] S. Chang, N. Weiner, I. Yavin, arXiv:1007.4200; N. Weiner, these proceedings.

[28] J. Kopp, V. Niro, T. Schwetz, J. Zupan, Phys. Rev. D80, 083502 (2009) [arXiv:0907.3159]; these proceedings. 